\title{
Antenna Reconfiguration Controller for 5G Communication Systems
}

\author{
Ayhan Yazgan*1 \\ Accepted $3^{\text {rd }}$ September 2016
}

\begin{abstract}
In this study, an Antenna Reconfiguration Controller (ARC) is presented. This controller is an electronic circuit based on 8 bit microprocessor and can be easily placed in the communication hardware platform. Proposed controller circuit can be used to reconfigure the antenna frequency, pattern or polarization for the possible $5 \mathrm{G}$ communication requirements. Designed ARC has the ability of switching selected PIN diodes or optical switches in order to obtain the desired frequency bands. Frequency reconfiguration of a monopole antenna experiment has also been conducted to prove the proposed ARC reliability. According to the measurement results the monopole antenna frequency has successfully been reconfigured from $900 \mathrm{MHz}$ (when the PIN diode is OFF) to $582 \mathrm{MHz}$ (when the PIN diode is $\mathrm{ON}$ ).
\end{abstract}

Keywords: Antenna reconfiguration, cognitive radio, $5 G$ communication

\section{Introduction}

Recently high speed communication systems have been attracting more and more attention by researchers. Undoubtedly, like a supply and demand balance, the necessity of the high speed communication keeps increasing. Since the available electromagnetic spectrum is limited, it is a good idea to enhance the spectrum utilization efficiency. One of the efficient methods is cognitive radio [1] which has been found a promising method by researchers for next generation communication systems such as 5G [2]. Cognitive radio includes different subsystems and scenarios to improve the spectrum efficiency. Its basic idea is to utilize the unused spectrum instantly whether it is licensed or not by different communication systems. For this reason, the active communication band may be changed by the operator in the limited range. As long as primary users do not sense the second ones, this system is a very good way to improve the spectrum efficiency. On the other hand, switching the communication frequency affects the antenna performance badly. In this point, in order to cover different frequency bands with high antenna gain, the reconfiguration process is used. This is a reasonable alternative solution instead of using several antennas which is not efficient considering the size restriction situation. Moreover, beside frequency reconfiguration, different properties of the antennas can be reconfigured such as antenna pattern or polarization [3, 4]. Several reconfiguration methods are used in literature [5-7]. However, electronic and optical reconfigurations are very topical in the literature. Both methods are being studied by universities and companies. Both have advantages and disadvantages relative to each other. For example, using an optical switch reduce the disturbance effect on the radiation pattern efficiently. However, in this case, a suitable laser and fiber cables are needed. This type switches are not easy to be inserted into the antenna body and expensive too. Another disadvantage is the power efficiency which is low. On the other hand, using an electronic switch some disturbances may occur due to the current carrying copper cables from ARC to the PIN diode inserted on the antenna surface. In this situation, keeping

Electrical-Electronics Engineering Department, Engineering Faculty, Karadeniz Technical University, 61080, Trabzon/Turkey

* Corresponding Author: Email: ayhanyazgan@ktu.edu.tr Note: This paper has been presented at the $3^{\text {rd }}$ International Conference on Advanced Technology \& Sciences (ICAT'16) held in Konya (Turkey), September 01-03, 2016. the current low may reduce the disturbance effect. Moreover, the experiment is easy, efficient and cheap comparing to the optical ones. MEMs switches are also used for reconfiguration process in literature. However MEMs are not used in this experiment. In this study, electronic reconfiguration is conducted with a high speed PIN diode which can be easily found commercially.

In the fabrication process, an electronic circuit based on 8 bit microprocessor is designed for antenna frequency reconfiguration purpose. After the design process, the Antenna Reconfiguration Controller (ARC) is fabricated and tested. The designed ARC has the capability of controlling 64 electronic or optical switches on the antenna surface. Although it supports up to 64 switches, when all the switches are used, it may increase the complexity and disturbance effect considering an electronic switching system which uses PIN diodes or MEMs switches mostly. For this reason, in the testbed, for the sake of simplicity, a monopole antenna is reconfigured using the fabricated ARC with only one PIN diode. As expected, two different resonant frequencies are obtained for a single monopole antenna.

For a complex antenna structure, more than one switch may be needed. In this case, a look-up table which contains all situations may be created. Another option is to write an optimization algorithm to find the minimum number of switches in order to cover the desired frequency band. For this reason, a microprocessor is always necessary for this kind of complex applications such as cognitive radio. For the cognitive radio process which is a possible network in $5 \mathrm{G}$ communication systems, the instant channel state information (CSI) is necessary in order to focus on the available frequency band. In this situation, a sensing antenna should be inserted into the system so as to scan and figure out the suitable frequency bands [8]. The sensing antennas are studied in literature and mostly selected as wide band antennas in order to scan maximum available electromagnetic spectrum. However sensing antennas are beyond the scope of this paper. In this study, frequency reconfiguration process is successfully fulfilled for a monopole antenna. On the other hand, it should be noted that, since the pattern or polarization reconfigurations have also the potential of increase the spectrum efficiency, these reconfiguration options may be needed for the future communication systems.

This paper is an expanded version of the previously published conference paper in ICAT 2016 [9], and it is organized as follows. In Section 2, designed and fabricated ARC is given. In Section 3, reconfiguration process is detailed with a monopole antenna example. In Section 4, possible intelligent designs are 


\section{Antenna Reconfiguration Controller (ARC)}

In Fig.1, the fabricated ARC is given. Parallel cables are connected to one port of the 8 bit microprocessor. LEDs are inserted for testing the signalization correctly. In order to test the ARC, a monopole antenna is designed with one high speed PIN diode as an electronic switch. The aim of this testbed is to use a monopole antenna operating in two different frequencies. The monopole size can be altered to obtain the desired frequency band. However the bandwidth of the antenna does not change is this method. 16F series 8 bit Microchip processor is selected for the ARC. As a high speed PIN diode, BAP63-02 is chosen from NXP semiconductor. Several ports are intentionally located in the $\mathrm{PCB}$ of the ARC so as to meet future possible purposes.

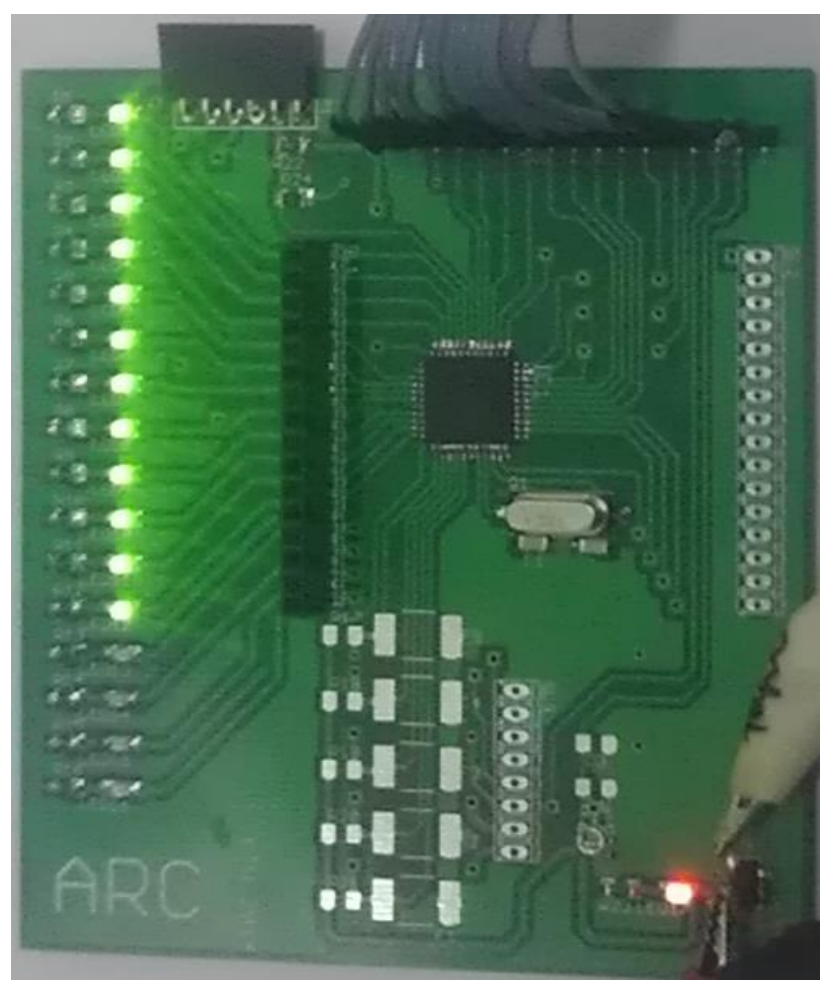

Figure.1. Fabricated Antenna Reconfiguration Controller

\section{Monopole Antenna Reconfiguration}

In this section, a monopole antenna reconfiguration is experimentally studied with the designed controller. ARC activates and deactivates the switch which is inserted on the antenna surface in the antenna fabrication process. Different kind of printed antennas may be reconfigured using the designed ARC. However, a simple monopole antenna is studied for the sake of simplicity. The reconfiguration process can be displayed by the connected LCD as given in Fig.2. In this study, only one switch is used and inserted on the antenna surface. However, if the number of switches needs to be increased, in this case, a look up table may be designed and inserted into the microprocessor by software as discussed above. A $5 \mathrm{~V}$ power supply is also seen in the testbed which is given in Fig.2. The simulated and designed monopole antenna is given in Fig.3.a. In Fig.3.b the PCB layout is given. The resistors and inductors are used to maintain the proper current flowing. According to the measurements, $2 \mathrm{~mA}$ was enough to switch the PIN diode from OFF to ON position.

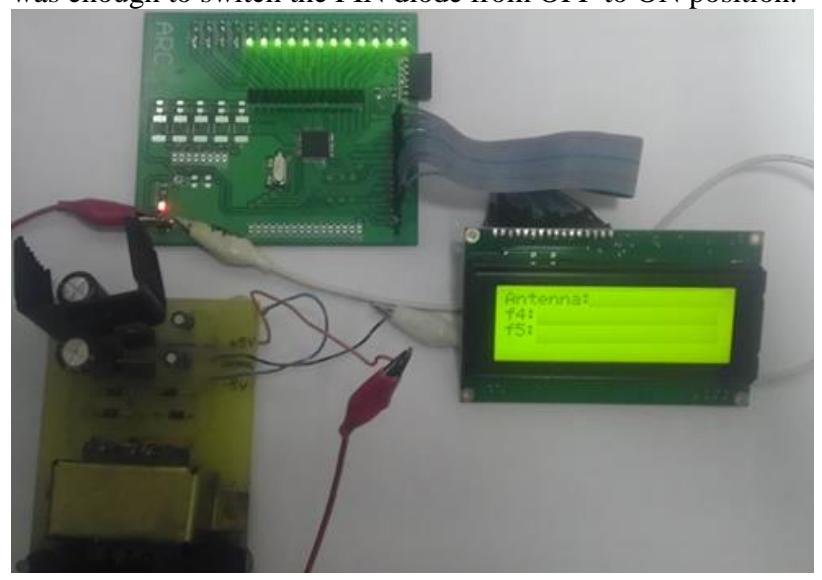

Figure2. Antenna test process

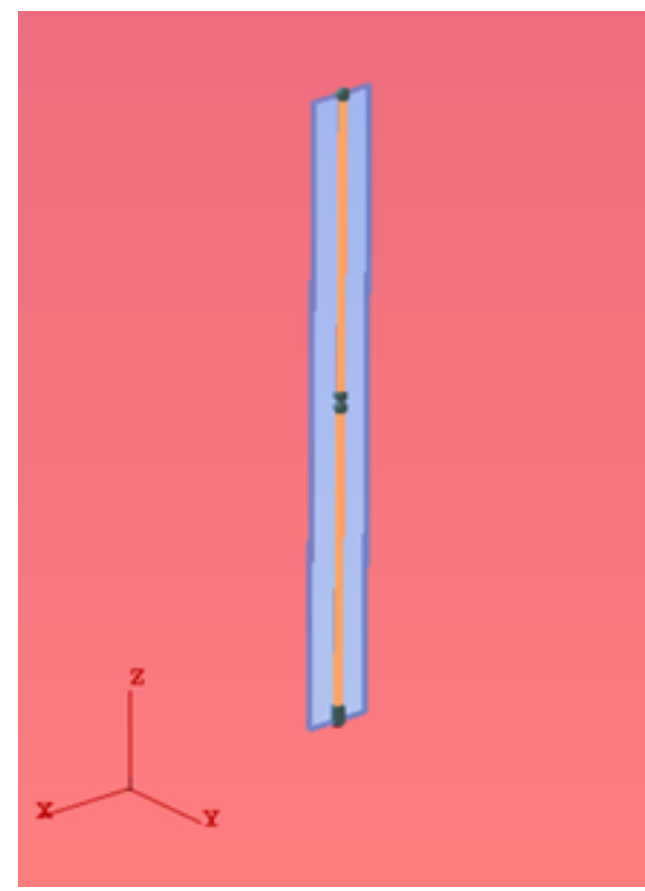

(a)

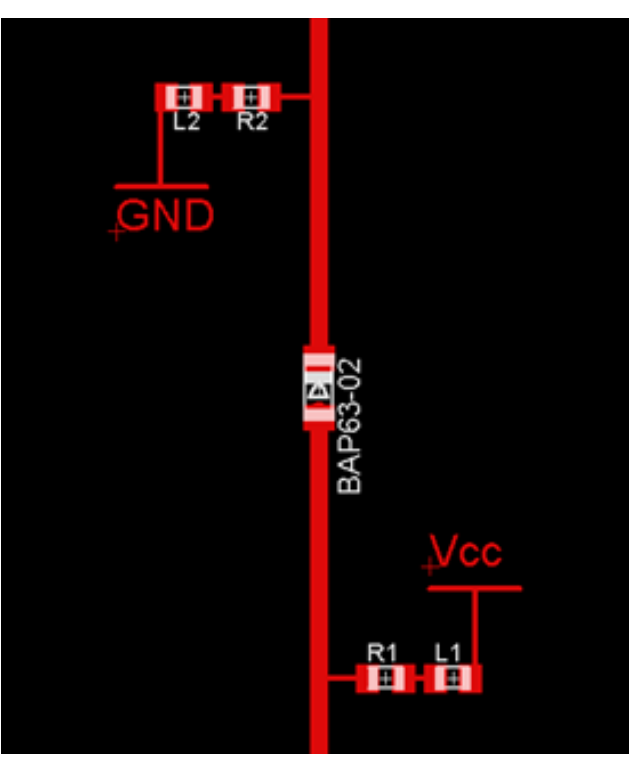

(b)

Figure.3. The simulation (a) and PCB (b) of the designed monopole antenna 


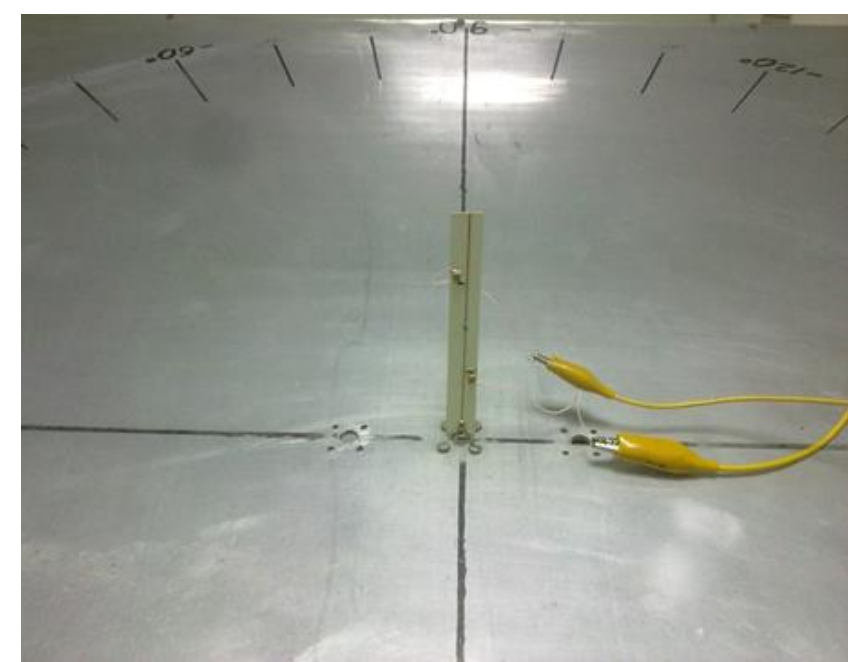

Figure.4. Monopole antenna reconfiguration test

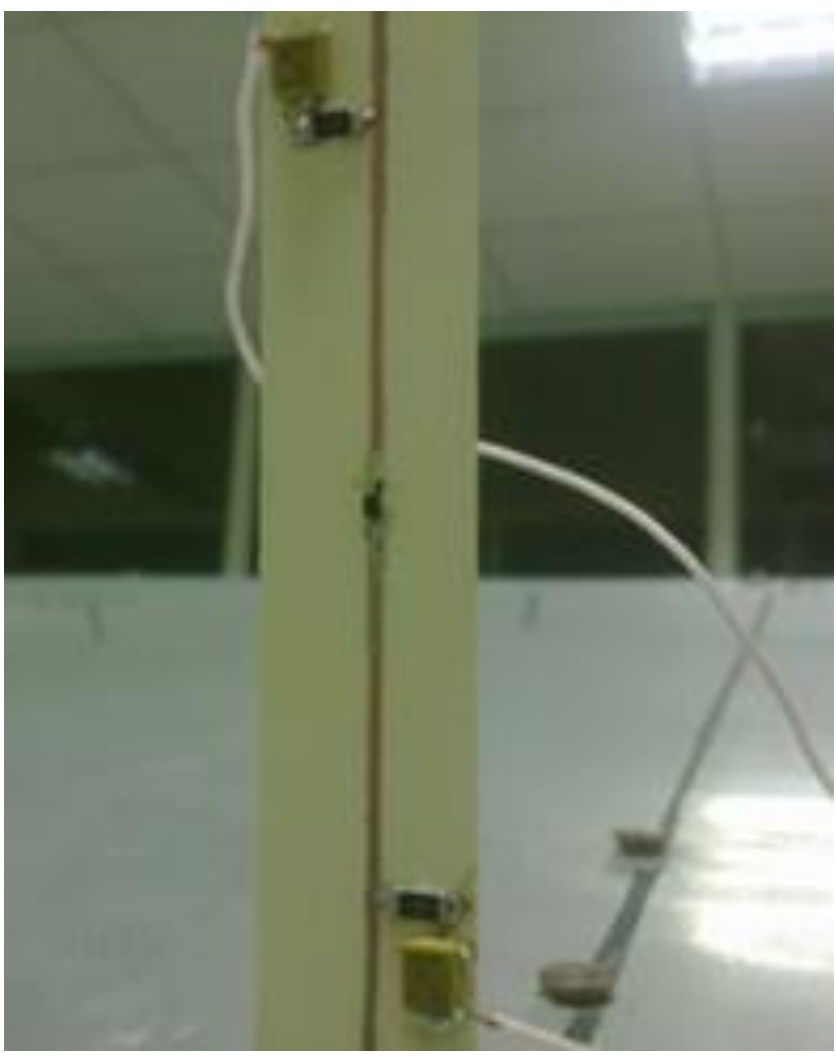

Figure.5. Monopole antenna under test

In Fig.4 and Fig.5, the fabricated antenna is given. The antenna is located on the circular ground plane as seen in the Figures. Antenna has special inductors, capacitors and resistors for the reconfiguration process. These electronic components and switching elements should be selected carefully since their electromagnetic effects can alter the resonance frequency [10]. Monopole antenna length is $100 \mathrm{~mm}$ and it is printed on the Rogers 4350 substrate $(\varepsilon r=3.48)$. According to the results, when the switch is turned on the resonance frequency of the antenna is measured as $582 \mathrm{MHz}$. Conversely, when the switch is turned off, the antenna resonance frequency is measured as $900 \mathrm{MHz}$ since dimension of the active wire decreases.

\section{Discussion}

In this study, the resonant frequencies were not intentionally selected. The goal was to test the reconfiguration hardware (ARC). However, a communication system operator should select these frequencies by using the CSI. In this case, the available channels or frequency bands may be determined and the system may focus on these special bands. As an example, the electromagnetic spectrum in $0-3 \mathrm{GHz}$ band was measured to see the available frequency bands in the communication laboratory of Karadeniz Technical University. The result is given in Fig.6. Here the frequency span is $3 \mathrm{GHz}$ and one horizontal square is $300 \mathrm{MHz}$. According to the measurement, for the moment when the samples were taken, the available frequency bands were between $1.22 \mathrm{GHz}$ to $1.78 \mathrm{GHz}$ and $2.5 \mathrm{GHz}$ to $3 \mathrm{GHz}$. Therefore, to minimize the frequency interference from environment, these frequency bands should be assigned for the $5 \mathrm{G}$ or cognitive radio communication systems. One may want to increase the resolution of the available bands by decreasing the focused bandwidth. In this case, the number of switches or the type of the antennas may be reorganized to meet this requirement.

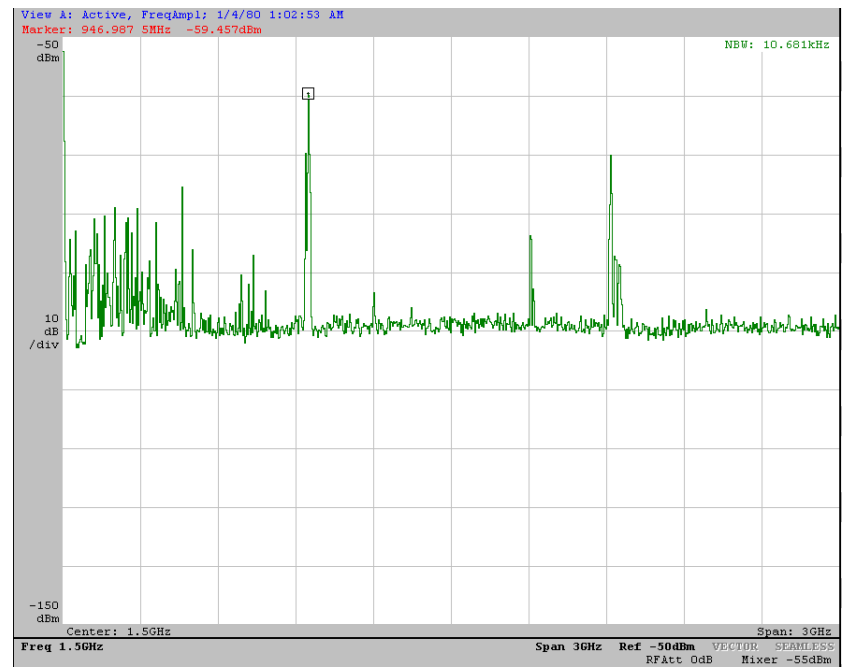

Figure.6. Spectrum measurement $0-3 \mathrm{GHz}$

\section{Conclusion}

Since it is an inevitable part of the cognitive radio which is suggested as possible 5G networks in literature, an antenna reconfiguration controller (ARC) was designed, fabricated and tested. In order to prove the reliability of the controller, a monopole antenna which is controlled by the ARC was fabricated and operated for two different frequencies. Electronic switching was used by inserting a PIN diode on the antenna surface. Using the fabricated ARC, reconfiguration process could be applied up to 64 switches. Increasing the number of switches may increase the scanned frequency resolution. However, considering the computational and hardware complexity it is clear that there is a trade-off. Therefore, optimum number of switches should be inserted on the antenna surfaces. Fabricated ARC performed well while switching the monopole antenna from $900 \mathrm{MHz}$ to 582 $\mathrm{MHz}$ and vice versa. 


\section{References}

[1] Mitola J., Maguire G. O., Cognitive radio: making software radios more personal, IEEE Personal Communications, Vol.6, no.4, pp.13-18, 1999.

[2] Hong X, Wang J., Wang C-X, Shi J., Cognitive radio in 5G: a perspective on energy-spectral efficiency trade-off, IEEE Communications Magazine, Vol.52, No. 7, pp.46-53, 2014.

[3] Aboufoul T., Alomainy A. ve Parini C., Polarization Reconfigurable Ultrawideband Antenna for Cognitive Radio Applications, Microwave and Optical Technology Letters, 55,3 (2013) 501-506.

[4] Aboufoul T., Shoaib I., Alomainy A. ve Xiaodong C. Pattern Reconfigurable Planar UWB Antenna Array for Future Cognitive Radio Portable Devices, Antennas and Propagation Conference (LAPC), Kasim 2012, Loughborough, Bildiriler Kitab1: 1-4.

[5] Tawk Y., Albrecht A. R., Hemmady S., Balakrishnan G., Christos G. ve Christodoulou C. G., Optically Pumped Frequency Reconfigurable Antenna Design, IEEE Antennas and Wireless Propagation Letters, 9 (2010) 280-283.

[6] Cai Y., Guo Y. J. ve Bird T. S., A Frequency Reconfigurable Printed Yagi-Uda Dipole Antenna for Cognitive Radio Applications, IEEE Transactions on Antennas and Propagation, 60,6 (2012) 2905-2912.

[7] Yazgan A., Kaya H., Çavdar İ.H., "Optically Reconfigurable Sierpinski Fractal Antennas For Rof Based Communication Systems", Telecommunication Systems, vol.59, no.4, pp.453-461, 2015.

[8] Zhang R., Lim T. L., Liang Y. C. ve Zeng Y., MultiAntenna Based Spectrum Sensing For Cognitive Radios a Glirt Approach, IEEE Transactions on Communication, 58,1 pp. 84-88, 2015.

[9] Yazgan A, "Microprocessor Based Antenna Reconfiguration Controller For 5G Communication Systems", 3rd International Conference on Advanced Technology \& Sciences (ICAT'16), pp.583-585, Konya, Turkey.

[10] Yazgan A., Çavdar İ.H., Kaya H., "The Effect Of Pin Diode Characteristics On The Antenna Reconfiguration Part Of Cognitive Radio", 38th International Conference on Telecommunications and Signal Processing (TSP), Prag, CEK CUM., 9-11, pp.191-194, 2015. 